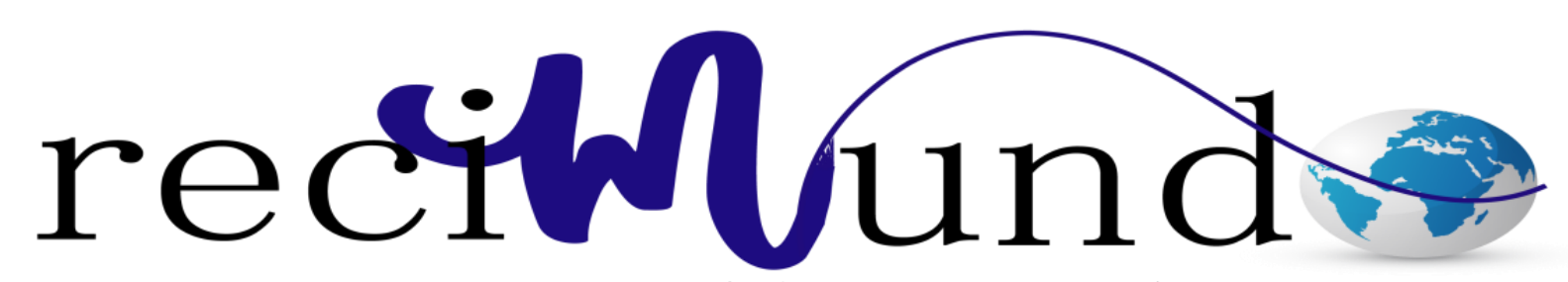

Revista Científica Mundo de la Investigación y el Conocimiento

Johanna Denys Suarez Orrala ${ }^{a}$; Jenniffer Katherine Molina Peñaherrera ${ }^{\text {b; }}$ Sheyla

Tamara Luna Martillo ${ }^{c}$; Geovanny Gabriel Garcia Cox ${ }^{d}$

Beneficios de la terapia renal continúa en pacientes críticos

Benefits of continuous renal therapy in critical patients

Revista Científica Mundo de la Investigación y el Conocimiento. Vol. 3 núm.3. Esp., noviembre, ISSN: 2588-073X, 2019, pp. 316-332

DOI: $10.26820 /$ recimundo/3.(3.Esp).noviembre.2019.316-332

URL: http://recimundo.com/index.php/es/article/view/610

Código UNESCO: 3205 Medicina Interna

Tipo de Investigación: Artículo de Revisión

(C) RECIMUNDO; Editorial Saberes del Conocimiento, 2019

Recibido: 15/09/2019

Aceptado: 23/10/2019

Publicado: 30/11/2019

Correspondencia: jdso_md@yahoo.com

a. Médico; Investigadora Independiente; Guayaquil, Ecuador; jdso_md@yahoo.com

b. Médico; Investigadora Independiente; Guayaquil, Ecuador; jenny1428_kath@ hotmail.com

c. Médico; Investigadora Independiente; Guayaquil, Ecuador; sheytalumar@ hotmail.com

d. Médico; Investigador Independiente; Guayaquil, Ecuador; geovanny_gc@yahoo.es 


\title{
Beneficios de la terapia renal continúa en pacientes críticos
}

\begin{abstract}
Vol. 3, núm. 3 Esp., (2019)
Johanna Denys Suarez Orrala; Jenniffer Katherine Molina Peñaherrera; Sheyla Tamara Luna Martillo; Geovanny Gabriel Garcia Cox
\end{abstract}

\section{RESUMEN}

La terapia de reemplazo renal continua (TRRC) es ahora el pilar de soporte de órganos renal en el estado crítico. A medida que nuestra comprensión de la entrega TRRC y su impacto en los resultados del paciente mejora hay un enfoque en la investigación de los beneficios potenciales de los tratamientos, el paciente específico adaptados a las necesidades dinámicas que se encuentran. Se revisan dosis TRRC, el calendario, la gestión de fluidos, la selección de membrana, la anticoagulación y acceso vascular: los estudios más recientes hasta la fecha que investigan aspectos de prescripción de TRRC que pueden individualizarse. El uso de diferentes dosis de TRRC carecen de pruebas convencional de alta calidad e importantes estudios revelan variación en la evaluación de la entrega de la dosis. La investigación revela pruebas contradictorias para los clínicos para distinguir qué pacientes se beneficiarían de 'espera vigilante' frente a la iniciación temprana de TRRC. A la vez que la dosificación dinámica de TRRC y manejo de fluidos utilizando TRRC son difíciles de investigar. Del mismo modo, la individualización de la elección de la membrana es en gran parte experimental. Los médicos tienen evidencia limitada para individualizar la prescripción de TRRC. Para desarrollar esto, se debe entender las necesidades de apoyo renal para los pacientes individuales, como el desequilibrio electrolítico, la sobrecarga de líquidos o aclaramiento de mediadores inflamatorios sistémicos que permitan dirigir a estas anormalidades en los ensayos aleatorios adecuadamente diseñados.

Palabras clave: Lesión renal aguda; Terapia de reemplazo renal continua; Cuidados críticos. 


\section{Beneficios de la terapia renal continúa en pacientes críticos}

Vol. 3, núm. 3 Esp., (2019)

Johanna Denys Suarez Orrala; Jenniffer Katherine Molina Peñaherrera; Sheyla Tamara Luna Martillo; Geovanny Gabriel Garcia Cox

\section{ABSTRACT}

Continuous renal replacement therapy (CRRT) is now the supporting pillar of renal organs in the critical state. As our understanding of TRRC delivery and its impact on patient outcomes improves there is a focus on the investigation of the potential benefits of treatments, the specific patient adapted to the dynamic needs that are encountered. TRRC doses, timing, fluid management, membrane selection, anticoagulation and vascular access are reviewed: the most recent studies to date investigating aspects of CRR prescription that can be individualized. The use of different doses of CRRT lacks high quality conventional tests and important studies reveal variation in the evaluation of dose delivery. The research reveals conflicting evidence for clinicians to distinguish which patients would benefit from 'watchful waiting' versus early initiation of CRRT. While dynamic dosing of CRRT and fluid management using CRRT is difficult to investigate. Similarly, the individualization of the membrane choice is largely experimental. Doctors have limited evidence to individualize the prescription of CRRT. To develop this, the needs of renal support for individual patients, such as electrolyte imbalance, fluid overload or clearance of systemic inflammatory mediators that allow to address these abnormalities in properly designed randomized trials, must be understood.

Keywords: Acute renal injury; Continuous renal replacement therapy; Critical care. 


\title{
Beneficios de la terapia renal continúa en pacientes críticos
}

\begin{abstract}
Vol. 3, núm. 3 Esp., (2019)
Johanna Denys Suarez Orrala; Jenniffer Katherine Molina Peñaherrera; Sheyla Tamara Luna Martillo; Geovanny Gabriel Garcia Cox
\end{abstract}

\section{Introducción.}

A nivel mundial, la terapia de reemplazo renal continua (TRRC) forma la piedra angular de la atención de apoyo para los pacientes críticamente enfermos con lesión renal aguda grave (AKI) (Kolhe, Muirhead, Wilkes, \& SR., 2015). Históricamente, la evidencia informa que la prescripción y entrega de TRRC se ha derivado de los estudios en los que los aspectos de entrega TRRC han sido investigados en el supuesto subyacente de que la prescripción 'óptima' de TRRC será el mismo en todos los pacientes en todos los puntos durante las necesidades individuales de terapia de reemplazo renal. Por lo tanto, mientras que los estudios clínicos proporcionan información valiosa para guiar a los estándares de base de restos en la entrega de TRRC aun permanecen inciertos la mejor forma de adaptar y personalizar la entrega TRRC a las necesidades individuales del paciente. Por ejemplo, hace 10 años dos estudios de referencia (Ronco, Bellomo, \& Homel, 2000) establecieron una dosis efectiva para TRRC en pacientes críticamente enfermos con IRA, sin embargo, sigue existiendo una necesidad en curso para investigar una terapia individualizada para satisfacer las necesidades de los escenarios clínicos específicos, incluyendo los subtipos de AKI. Para este fin, la reunión de la Conferencia de Consenso Iniciativa de Calidad de Enfermedades 17a aguda en Asiago, Italia (10-13 de junio de 2016) 'Precisión renal continuo en terapia de reemplazo' esbozó un programa de investigación extensa para mejorar, entregar y supervisar una receta dinámica TRRC diseñada para satisfacer el soluto individual y los requerimientos de líquidos de los pacientes (Bagshaw, Chakravarthi, \& Ricci, 2016). Con estas recomendaciones en mente, evaluamos la evidencia más reciente que podría apoyar precisión TRRC a través de la individualización de su prescripción y entrega (Tabla 1). 


\title{
Beneficios de la terapia renal continúa en pacientes críticos
}

Vol. 3, núm. 3 Esp., (2019)

Johanna Denys Suarez Orrala; Jenniffer Katherine Molina Peñaherrera; Sheyla Tamara Luna Martillo; Geovanny Gabriel Garcia Cox

Sistema renal

\section{PUNTOS CLAVE}

\begin{abstract}
Buena evidencia para guiar la individualización de TRRC se carece actualmente como tal, no queda mucha variabilidad en la entrega de TRRC.

El uso de diferentes dosis de TRRC carecen de pruebas convencionales de alta calidad y hay pruebas contradictorias para los clínicos para distinguir qué pacientes se beneficiarían de 'espera vigilante' frente a la iniciación temprana de TRRC.

Tanto la dosificación dinámica TRRC y manejo de fluidos de precisión utilizando TRRC son difíciles de investigar y actualmente solo la observación de datos soporta la individualización de las recetas.
\end{abstract}

Para el desarrollo de este proceso investigativo, se plantea como metodología la encaminada hacia una orientación científica particular que se encuentra determinada por la necesidad de indagar en forma precisa y coherente una situación, en tal sentido (Davila, 2015) define la metodología "como aquellos pasos previos que son seleccionados por el investigador para lograr resultados favorables que le ayuden a plantear nuevas ideas". (p.66).

Lo citado por el autor, lleva a entender que el desarrollo de la acción investigativa busca simplemente coordinar acciones enmarcadas en una revisión bibliográfica con el fin de complementar ideas previas relacionadas al beneficio de terapia renal continua en pacientes críticos a través de una revisión de literatura, para así finalmente elaborar un cuerpo de consideraciones generales que ayuden a ampliar el interés propuesto.

\section{Tipo de Investigación.}

Dentro de toda práctica investigativa, se precisan acciones de carácter metodológico mediante las cuales, se logra conocer y proyectar los eventos posibles que la determinan, así como las características que hacen del acto científico un proceso interactivo ajustado a una 


\title{
Beneficios de la terapia renal continúa en pacientes críticos
}

\author{
Vol. 3, núm. 3 Esp., (2019) \\ Johanna Denys Suarez Orrala; Jenniffer Katherine Molina Peñaherrera; Sheyla Tamara Luna \\ Martillo; Geovanny Gabriel Garcia Cox \\ realidad posible de ser interpretada. En este sentido, se puede decir, que la presente investigación \\ corresponde al tipo documental, definido por Castro (2016), "se ocupa del estudio de problemas \\ planteados a nivel teórico, la información requerida para abordarlos se encuentra básicamente en \\ materiales impresos, audiovisuales y /o electrónicos". (p.41).
}

En consideración a esta definición, la orientación metodológica permitió la oportunidad de cumplir con una serie de actividades inherentes a la revisión y lectura de diversos documentos donde se encontraron ideas explicitas relacionadas con los tópicos encargados de identificar a cada característica insertada en el estudio. Por lo tanto, se realizaron continuas interpretaciones con el claro propósito de revisar aquellas apreciaciones o investigaciones propuestas por diferentes investigadores relacionadas con el tema de interés, para luego dar la respectiva argumentación a los planteamientos, en función a las necesidades encontradas en la indagación.

\section{Fuentes Documentales}

El análisis correspondiente a las características que predomina en el tema seleccionado, llevan a incluir diferentes fuentes documentales encargadas de darle el respectivo apoyo y en ese sentido cumplir con la valoración de los hechos a fin de generar nuevos criterios que sirven de referencia a otros procesos investigativos. Para (CASTRO, TÉCNICAS DOCUMENTALES. MÉXICO., 2016) las fuentes documentales incorporadas en la investigación documental o bibliográfica, "representa la suma de materiales sistemáticos que son revisados en forma rigurosa y profunda para llegar a un análisis del fenómeno". (p.41). Por lo tanto, se procedió a cumplir con la realización de una lectura previa determinada para encontrar aquellos aspectos 


\section{Beneficios de la terapia renal continúa en pacientes críticos}

Vol. 3, núm. 3 Esp., (2019)

Johanna Denys Suarez Orrala; Jenniffer Katherine Molina Peñaherrera; Sheyla Tamara Luna Martillo; Geovanny Gabriel Garcia Cox

estrechamente vinculados con el tema, con el fin de explicar mediante un desarrollo las respectivas apreciaciones generales de importancia.

Técnicas para la Recolección de la Información

La conducción de la investigación para ser realizada en función a las particularidades que determinan a los estudios documentales, tiene como fin el desarrollo de un conjunto de acciones encargadas de llevar a la selección de técnicas estrechamente vinculadas con las características del estudio. En tal sentido, (Bolívar, Investigación Documental. México., 2015), refiere, que es "una técnica particular para aportar ayuda a los procedimientos de selección de las ideas primarias y secundarias". (p. 71).

Por ello, se procedió a la utilización del subrayado, resúmenes, fichaje, como parte básica para la revisión y selección de los documentos que presentan el contenido teórico. Es decir, que mediante la aplicación de estas técnicas se pudo llegar a recoger informaciones en cuanto a la revisión bibliográfica de los diversos elementos encargados de orientar el proceso de investigación. Tal como lo expresa, (Bolívar, Investigación Documental. México., 2015) "las técnicas documentales proporcionan las herramientas esenciales y determinantes para responder a los objetivos formulados y llegar a resultados efectivos" (p. 58). Es decir, para responder con eficiencia a las necesidades investigativas, se introdujeron como técnica de recolección el método inductivo, que hizo posible llevar a cabo una valoración de los hechos de forma particular para llegar a la explicación desde una visión general. 


\title{
Beneficios de la terapia renal continúa en pacientes críticos
}

\author{
Vol. 3, núm. 3 Esp., (2019) \\ Johanna Denys Suarez Orrala; Jenniffer Katherine Molina Peñaherrera; Sheyla Tamara Luna \\ Martillo; Geovanny Gabriel Garcia Cox
}

Asimismo, se emplearon las técnicas de análisis de información para la realización de la investigación que fue ejecutada bajo la dinámica de aplicar diversos elementos encargados de determinar el camino a recorrer por el estudio, según, (Bolívar, Investigación Documental. México., 2015) las técnicas de procesamiento de datos en los estudios documentales "son las encargadas de ofrecer al investigador la visión o pasos que debe cumplir durante su ejercicio, cada una de ellas debe estar en correspondencia con el nivel a emplear" (p. 123). Esto indica, que para llevar a cabo el procesamiento de los datos obtenidos una vez aplicado las técnicas seleccionadas, tales como: fichas de resumen, textual, registros descriptivos entre otros, los mismos se deben ajustar al nivel que ha sido seleccionado.

\section{Resultados.}

Individualización de las dosis

Las dosis de TRRC se calcula comúnmente como el ultrafiltrado en la hemofiltración venosa continua, entregado volumen de dializado en la hemodiálisis venosa continua (con tasas de flujo de dializado lentas mucho menor que el caudal de sangre) y una combinación de ambos para hemodiafiltración continuo venosa. El volumen resultante a menudo expresada como velocidad de flujo del efluente en mililitros por hora o mililitros por kilogramo de peso corporal por hora $(\mathrm{ml} / \mathrm{kg} / \mathrm{h})$ es un sustituto razonable para la liquidación de pequeños topo cules tales como urea. Basándose en los resultados de diversos pernos prisioneros, la (Kidney Disease Improving Global Outcomes, 2012) recomienda una dosis objetivo de $20-25 \mathrm{ml} / \mathrm{kg} / \mathrm{h}$, sino que también permite el ajuste dinámico de la dosificación para satisfacer las necesidades de una enfermedad aguda. Aunque no hay evidencia que demuestre que la dosificación dinámica de 


\section{Beneficios de la terapia renal continúa en pacientes críticos}

Vol. 3, núm. 3 Esp., (2019)

Johanna Denys Suarez Orrala; Jenniffer Katherine Molina Peñaherrera; Sheyla Tamara Luna Martillo; Geovanny Gabriel Garcia Cox

TRRC mejora los resultados a corto o largo plazo, la gestión individualizada es inherentemente difícil de investigar. Incluso en los estudios aleatorios de establecimiento de dosis algunas recomendaciones fueron excluidas tales como aquellos con exceso de peso o la enfermedad avanzada renal crónica (ERC) y la variación de los grupos importantes de los pacientes de protocolo se produjo en los casos en que las anormalidades metabólicas eran persistentes en la receta original. Recomendaciones de dosis importantes actuales no cubren el ajuste practicado comúnmente de TRRC de dosis en el contexto de las anormalidades metabólicas agudas graves o la recuperación y la estabilidad clínica. Además, para ello, la necesidad de marcadores adicionales de depuración de solutos y por lo tanto la eficacia de TRRC entregada ha sido puesta de relieve por los grupos de expertos en consenso. Una sugerencia ha sido la de refinación de dosis de evaluación basada en la tasa de efluentes a un flujo específico de TRRC, estandarizado Kt / V (aclaramiento de urea en el tiempo indexado a volumen de distribución de urea) (Clark, Leblanc, Ricci, \& Ronco, 2017). Sin embargo, mientras que más suena en teoría no está claro si la complejidad adicional es clínicamente útil en la cabecera. 


\title{
Beneficios de la terapia renal continúa en pacientes críticos
}

\author{
Vol. 3, núm. 3 Esp., (2019) \\ Johanna Denys Suarez Orrala; Jenniffer Katherine Molina Peñaherrera; Sheyla Tamara Luna \\ Martillo; Geovanny Gabriel Garcia Cox
}

Tabla 1. Los dominios de terapia sustitutiva renal continua con receta y áreas de individualización

\begin{tabular}{|c|c|c|c|}
\hline Dominios de prescripción & Parámetro & $\begin{array}{l}\text { Situaciones potenciales para } \\
\text { individualización }\end{array}$ & Peligros potenciales de la prescripción \\
\hline Dosis & velocidad de flujo de efluentes & $\begin{array}{l}\text { carga de solutos } \\
\text { Acidosis } \\
\text { alteraciones electrolíticas }\end{array}$ & $\begin{array}{l}\text { eliminación de los antibióticos } \\
\text { Desequilibrio } \\
\text { la pérdida de micronutrientes } \\
\text { Alcalosis }\end{array}$ \\
\hline \multicolumn{4}{|l|}{$\begin{array}{l}\text { Sincro } \\
\text { nizació }\end{array}$} \\
\hline & $\begin{array}{l}\text { Criterios para la apertura } \\
\text { terapia }\end{array}$ & $\begin{array}{l}\text { postoperatoria AKI } \\
\text { La sobrecarga de líquidos }\end{array}$ & $\begin{array}{l}\text { terapia inadecuada } \\
\text { No recuperación de la función renal }\end{array}$ \\
\hline $\begin{array}{l}\text { Ultrafiltración } \\
\text { Reemplazar fluido / } \\
\text { diálisis }\end{array}$ & $\begin{array}{l}\text { tasa de eliminación de líquido } \\
\text { composición de electrolito } \\
\text { Fluid }\end{array}$ & $\begin{array}{l}\text { La sobrecarga de líquidos } \\
\text { alteraciones electrolíticas }\end{array}$ & $\begin{array}{l}\text { La inestabilidad hemodinámica y lesión de } \\
\text { órganos } \\
\text { tarifas inapropiadas de corrección } \\
\text { Los errores clínicos }\end{array}$ \\
\hline Membrana & propiedades de adsorción & $\begin{array}{l}\text { Sepsis y sistémica } \\
\text { inflamación }\end{array}$ & $\begin{array}{l}\text { La eliminación de exógena y beneficiosa } \\
\text { sustancias endógenas (antibióticos, } \\
\text { albúmina, etc.) }\end{array}$ \\
\hline La anticoagulación & elección anticoagulante & $\begin{array}{l}\text { Diátesis hemorrágica, } \\
\text { La disfunción mitocondrial, } \\
\text { disfunción hepática }\end{array}$ & $\begin{array}{l}\text { El sangrado, pérdida de circuito, la } \\
\text { acumulación de citrato, } \\
\text { trombocitopenia inducida por heparina }\end{array}$ \\
\hline El acceso vascular & localización anatómica & $\begin{array}{l}\text { La mala vida útil del filtro } \\
\text { difíciles } \\
\text { anatomía vascular }\end{array}$ & $\begin{array}{l}\text { A corto y largo plazo las complicaciones } \\
\text { de la línea de } \\
\text { inserción }\end{array}$ \\
\hline
\end{tabular}

En general, nuestra capacidad de adaptar dosis de TRRC está limitada si no podemos garantizar dosis estandarizadas cuando se prescribe. En el estudio observacional prospectivo (Vesconi, Cruz, \& Fumagalli, 2009) la variabilidad significativa de la dosis administrada de TRRC se observó entre los pacientes durante el curso de los tratamientos dentro de los individuos. En varios estudios, la dosis TRRC suministrada ha demostrado ser 20-30\% por debajo de la prescrita con la pérdida de circuito no deseados y tiempo de inactividad filtro de la principal causa (Lyndon, Wille, \& Tolwani, 2012). Las mejoras en la tecnología de TRRC para ayudar a los clínicos en la entrega precisa de la dosis objetivo y mejorar dosis-evaluaciones se están haciendo disponibles como la compensación de dosis, el tiempo de inactividad. 


\section{Beneficios de la terapia renal continúa en pacientes críticos}

Vol. 3, núm. 3 Esp., (2019)

Johanna Denys Suarez Orrala; Jenniffer Katherine Molina Peñaherrera; Sheyla Tamara Luna Martillo; Geovanny Gabriel Garcia Cox

El concepto de la adaptación de la intensidad TRRC a las demandas de la enfermedad ha sido explorado por el análisis del efecto de la dosificación en categorías específicas de pacientes en estado crítico. Varios estudios no han podido demostrar ningún beneficio de dosis mayores de TRRC en pacientes con sepsis (Joannes-Boyau, Honore', \& Perez, 2013), resultados similares en meta-análisis de TRRC no fueron influenciados por el porcentaje de pacientes con sepsis o shock séptico que sugiere conclusiones generales sobre la intensidad de TRRC pertenecen a un subgrupo sepsis. Del mismo modo, los pacientes con insuficiencia hepática representan un grupo con potencial para beneficiarse de una mayor intensidad de TRRC, con datos asociados a la reducción de amoníaco con la mejora de los resultados clínicos. En adición, los pacientes con disfunción hepática prescritos con una dosis más alta a menudo fracasado en lograr la dosis prescrita y experimentaron una mayor tasa de hipofosfatemia. Un estudio pequeño, prospectivo investiga dosis más alta de hemofiltración venosa continua (CVVH) en pacientes con quemaduras no mostró ninguna diferencia en la mortalidad en comparación con una dosis normal excepto en el subgrupo de pacientes con las quemaduras severas (You, Zhang, \& Luo, 2018). Del mismo modo en rabdomiólisis, en el que existe plausibilidad biológica para una mayor intensidad de TRRC para eliminar la mioglobina hay ensayos no suficientemente accionados controlados aleatorios (ECA) para proporcionar evidencia de la eficacia. Por lo tanto, sigue existiendo una falta de apoyo para una mayor intensidad de TRRC basado sólo en los grupos de pacientes distintos. Por un lado, esto puede sugerir que una talla única de aproximación a la intensidad de TRRC es el correcto, sin embargo, por otro lado, es posible que los factores individuales de los pacientes pueden ser más importantes que arquear categorías clínicas, que pueden variar mucho en el contexto clínico y gravedad. 


\title{
Beneficios de la terapia renal continúa en pacientes críticos
}

\author{
Vol. 3, núm. 3 Esp., (2019) \\ Johanna Denys Suarez Orrala; Jenniffer Katherine Molina Peñaherrera; Sheyla Tamara Luna \\ Martillo; Geovanny Gabriel Garcia Cox
}

Es importante destacar que, mientras que los beneficios de la dosis más alta de TRRC no están claras la presunción de que puede beneficiar a algunos pacientes deben ser templado en contra de la posibilidad de daño con mayor intensidad de tratamiento. Una colaboración reciente, meta-análisis de datos paciente individual ha demostrado un tiempo más largo a la cesación de la terapia de reemplazo renal en pacientes que reciben un régimen de mayor intensidad. El estudio incluyó tanto TRRC y modalidades intermitentes, pero el análisis de subgrupos mostró consistencias entre los grupos y, por tanto, hace hincapié en la falta de pruebas y potencial de daño de los regímenes TRRC más intensivos (Wang, Gallagher, \& Li, 2018). Además, la asociación de dosis más altas de TRRC con disturbios de electrolito ha vuelto cada vez más reconocidos como tiene el impacto de TRRC de otros factores importantes, tales como antibióticos y micronutrientes. Por lo tanto, la individualización de la prescripción TRRC tiene que ir acompañada de la individualización de otras terapias. Se ha expresado preocupación con respecto significativa bajo la dosificación de los antibióticos, a menudo uno de los tratamientos esenciales que se ofrecen a los pacientes críticamente enfermos y los aumentos en la probabilidad de resistencia y el fracaso del tratamiento llevando eventualmente a un número creciente de antibióticos que se utilizan.

El aclaramiento y la exigencia de micronutrientes, oligoelementos y vitaminas para los pacientes que reciben TRRC ha sido objeto de investigaciones recientes. (Kamel, Dave, \& Zhao, 2018) reporto $80 \%$ de los pacientes en un estudio retrospectivo para tener un nivel inferior a lo normal de al menos uno de micronutrientes. Existe poca orientación en cuanto a la mejor práctica para la prescripción de vitaminas para los pacientes que reciben TRRC. Del mismo modo, la investigación se está convirtiendo en el impacto de TRRC en la ingesta de calorías y las 


\section{Beneficios de la terapia renal continúa en pacientes críticos}

Vol. 3, núm. 3 Esp., (2019)

Johanna Denys Suarez Orrala; Jenniffer Katherine Molina Peñaherrera; Sheyla Tamara Luna Martillo; Geovanny Gabriel Garcia Cox

demandas metabólicas de los pacientes críticamente enfermos con una anticoagulación regional citrato-dextrosa y fluido de sustitución que contiene dextrosa en un circuito CVVH demostrado significativamente contribuyen a la ingesta de calorías

\section{Individualización del manejo de fluidos}

La declaración de consenso ADQI-17 sugiere precisión en la gestión de fluidos en TRRC debe incorporar efectos en el equilibrio de fluidos del paciente, el circuito de integridad y la composición de plasma (Murugan, Hoste, \& Mehta, 2016). Este proceso dinámico debe adaptarse a las exigencias del paciente y requiere un equilibrio entre la extracción neta de fluido para permitir tratamientos tales como antibióticos y la ingesta de líquidos netos para alcanzar los objetivos hemodinámicos. La asociación de la acumulación de fluido con la mortalidad está bien informada y es particularmente fuerte en el contexto de AKI. Un estudio retrospectivo reciente de resucitación soportada de los pacientes con sobrecarga de líquidos iatrogénica y de la mortalidad mostró cuando un equilibrio de líquidos mínimo estaba dirigido a 3 día usando diuréticos y / o RRT (Hjortrup, Haase, Wetterslev, \& Perner, 2016). Los estudios se centraron en el papel específico de TRRC en la eliminación de líquido en estos pacientes no están disponibles actualmente y un estudio piloto reciente prueba de una estrategia de eliminación de líquido incluyendo el uso de TRRC en la enfermedad crítica sugiere que será un reto para adquirir estos datos, ya que las tasas de inclusión eran muy bajas. La inestabilidad hemodinámica en o cerca del inicio de TRRC se ha asociado con resultados más pobres y puede limitar la eliminación de líquido. 


\title{
Beneficios de la terapia renal continúa en pacientes críticos
}

\author{
Vol. 3, núm. 3 Esp., (2019) \\ Johanna Denys Suarez Orrala; Jenniffer Katherine Molina Peñaherrera; Sheyla Tamara Luna \\ Martillo; Geovanny Gabriel Garcia Cox
}

Aunque hay muchos tipos diferentes de sustitución de fluidos en TRRC disponible con una gama de diferentes composiciones de electrolito y tampones, hay poca evidencia disponible para ayudar a guiar la selección de un clínico de reposición de líquidos en pacientes mientras que a menudo es de fabricación y protocolo en lugar de en el paciente específico. Alteraciones electrolíticas severas, en particular los de sodio en el suero, son una situación en la que la adaptación de prescripciones TRRC y soluciones se indica fuertemente para evitar el exceso de anomalías, los protocolos, sin embargo específicos siguen basándose en el asesoramiento de expertos y experiencia de pequeña series de casos con pocas perspectivas de pruebas de alta calidad se define convencionalmente estén disponibles para guiar a los médicos en esta importante área.

\section{Individualización de membrana}

Las modificaciones de las membranas utilizadas en TRRC ofrecen el potencial para mejorar la eliminación de una gama de compuestos potencialmente dañinos tales como los mediadores inflamatorios que se presentan en pacientes con AKI séptico.

Un reciente estudio in vitro (Malard, Lambert, \& Kellum, 2018). La investigación de las propiedades de adsorción variable de dispositivos avanzados incluyendo membrana Oxiris, pone de relieve su potencial para la eliminación de endotoxina y otros mediadores inflamatorios para modular la respuesta inmune en pacientes con sepsis, sin embargo, grandes ensayos clínicos posibles todavía no se han realizado. Alternativamente, en lugar de la adsorción, los altos filtros de corte permiten la eliminación de una amplia gama de mediadores de mayor peso molecular debido a su mayor tamaños de poro. Sin embargo, un ECA que investiga el tratamiento de los 


\section{Beneficios de la terapia renal continúa en pacientes críticos}

Vol. 3, núm. 3 Esp., (2019)

Johanna Denys Suarez Orrala; Jenniffer Katherine Molina Peñaherrera; Sheyla Tamara Luna Martillo; Geovanny Gabriel Garcia Cox

pacientes críticamente enfermos con corte alto membranas reveló ninguna reducción en la mortalidad o vasopresores (Liu, Mao, \& Kang, 2016)Estos datos apoyan el actual punto de vista de que las estrategias de individualización del uso de membranas en TRRC siguen siendo experimentales.

\section{Individualización de anticoagulación}

La anticoagulación regional con citrato está ahora reconocida como el modo de la anticoagulación de elección para TRRC, en base a estudios que demuestran la mejora de la vida del filtro, menos sangrado sistémica y por lo tanto una mejor prestación de tratamiento. Sin embargo, esto sigue siendo la recomendación que ha de apoyar evidencia de grandes estudios multicéntricos y la completacion de investigación entre los diferentes anticoagulantes para la Terapia de Reemplazo Renal.

\section{Conclusiones.}

Falta de desarrollo de pruebas de alta calidad para apoyar su uso sea más intensivo o antes de TRRC para AKI en poblaciones mixtos en estado crítico ha animado a los protocolos estandarizados para la prescripción y entrega de TRRC durante la enfermedad crítica. Sin embargo, en la práctica clínica, el uso y la entrega de TRRC se ha demostrado ser muy variable. En ausencia de pruebas positivas fuertes algunos clínicos individualizan la prescripción de TRRC sobre la base de intuición clínica, mientras que otros adoptan un enfoque liberal de la entrega TRRC. De hecho, muchos escenarios clínicos comunes caen fuera del alcance de la base de la evidencia disponible. Por lo tanto, el potencial existe prescripciones específicas a las 


\section{Beneficios de la terapia renal continúa en pacientes críticos}

Vol. 3, núm. 3 Esp., (2019)

Johanna Denys Suarez Orrala; Jenniffer Katherine Molina Peñaherrera; Sheyla Tamara Luna Martillo; Geovanny Gabriel Garcia Cox

necesidades de los pacientes y sus enfermedades, pero esto probablemente no se extiende a las definiciones amplias como séptica-AKI, que es en realidad muy heterogénea. Sin embargo, una buena evidencia de guía de individualización de la terapia actualmente se carece. Para desarrollar esto, tenemos que soportar el sub-requisito clave para el apoyo renal en un individuo, tales como el desequilibrio electrolítico o sobrecarga de líquidos, y para orientar estas anormalidades en los ensayos aleatorios adecuadamente diseñados. Por último, como la entrega de cualquier prescripción individualizada depende de la vida útil del filtro, la individualización de los aspectos operativos de TRRC tales como la posición del catéter y la anticoagulación, es un complemento clave para entregar de forma fiable una prescripción individualizada y para orientar estas anormalidades en los ensayos aleatorizados adecuadamente diseñados.

\section{Bibliografía.}

Bolívar, J. (2015). Investigación Documental. México: Pax.

Castro, J. (2016). Técnicas Documentales. México: Limusa.

Clark, W., Leblanc, M., Ricci, Z., \& Ronco, C. (2017). Quantification and dosing of renal \& replacement therapy in acute kidney injury: a reappraisal. Blood Purif, 44(2), 140-155.

Davila, A. (2015). Concepto de terminos cientificos. Caracas: Oasis.

Hjortrup, P., Haase, N., Wetterslev, J., \& Perner, A. (2016). Associations of hospital and patient characteristics with fluid resuscitation volumes in patients with severe sepsis: post hoc analyses of data from a multicentre randomised clinical trial. PLoS One, 11(5), 1-11.

Kamel, A., Dave, N., \& Zhao, V. (2018). Micronutrient alterations during con-tinuous renal replacement therapy in critically ill adults: a retrospective study. Nutr Clin Pract, 33(3), 439-446.

Kidney Disease Improving Global Outcomes. (2012). KDIGO clinical practice guide-line for acute kidney injury. Kidney Int Suppl, 2(1), 19-36. 


\section{Beneficios de la terapia renal continúa en pacientes críticos}

Vol. 3, núm. 3 Esp., (2019)

Johanna Denys Suarez Orrala; Jenniffer Katherine Molina Peñaherrera; Sheyla Tamara Luna Martillo; Geovanny Gabriel Garcia Cox

Kolhe, N., Muirhead, A., Wilkes, \& SR. (2015). National trends in acute kidney injury requiring dialysis in England between 1998 and 2013. Kidney Int, 88(5), 1161-1169.

Liu, C., Mao, Z., \& Kang, H. (2016). Regional citrate versus heparin anticoagulation for continuous renal replacement therapy in critically ill patients: a meta-analysis with trial sequential analysis of randomized controlled trials. Crit Care, 20(1), 1-144.

Lyndon, W., Wille, K., \& Tolwani, A. (2012). Solute clearance in CRRT: prescribed dose versus actual delivered dose. Nephrol Dial Transplant, 27(3), 952-956.

Malard, B., Lambert, C., \& Kellum, J. (2018). In vitro comparison of the adsorption of \& inflammatory mediators by blood purification devices. Intensive Care Med Exp, 6(1), 112.

Ronco, C., Bellomo, R., \& Homel, P. (2000). Effects of different doses in continuous venovenous haemofiltration on outcomes of acute renal failure: a prospective randomised trial. Lancet, 356(9223), 26-30.

Vesconi, S., Cruz, D., \& Fumagalli, R. (2009). Delivered dose of renal replacement therapy and mortality in critically ill patients with acute kidney injury. Crit Care, 13(2), 1-14.

Wang, Y., Gallagher, M., \& Li, Q. (2018). Renal replacement therapy intensity for \& acute kidney injury and recovery to dialysis independence. Nephrol Dial Transplant, 33(6), 1017-1024.

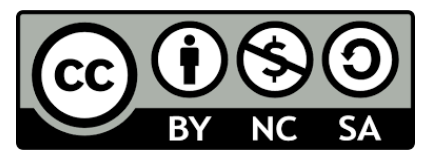

\section{RECONOCIMIENTO-NOCOMERCIAL-COMPARTIRIGUAL}

CC BY-NC-SA

ESTA LICENCIA PERMITE A OTROS ENTREMEZCLAR, AJUSTAR Y CONSTRUIR A PARTIR DE SU OBRA CON FINES NO COMERCIALES, SIEMPRE Y CUANDO LE RECONOZCAN LA AUTORÍA Y SUS NUEVAS CREACIONES ESTÉN BAJO UNA LICENCIA CON LOS MISMOS TÉRMINOS. 


\section{Beneficios de la terapia renal continúa en pacientes críticos}

Vol. 3, núm. 3 Esp., (2019)

Johanna Denys Suarez Orrala; Jenniffer Katherine Molina Peñaherrera; Sheyla Tamara Luna

Martillo; Geovanny Gabriel Garcia Cox 